\title{
The role for neutrophil extracellular traps in cystic fibrosis autoimmunity
}

\author{
Sladjana Skopelja, ${ }^{1}$ B. JoNell Hamilton, ${ }^{1}$ Jonathan D. Jones, ${ }^{2}$ Mei-Ling Yang, ${ }^{3}$ Mark Mamula, ${ }^{3}$ \\ Alix Ashare, ${ }^{1,4}$ Alex H. Gifford, ${ }^{4}$ and William F.C. Rigby ${ }^{1,2}$ \\ 'Department of Microbiology and Immunology and 'Division of Rheumatology, Department of Medicine, Geisel School of \\ Medicine at Dartmouth, Lebanon, New Hampshire, USA. ${ }^{3}$ Section of Rheumatology, Department of Internal Medicine, Yale \\ University School of Medicine, New Haven, Connecticut, USA. ${ }^{4}$ Division of Pulmonology, Department of Medicine, Geisel \\ School of Medicine at Dartmouth, Lebanon, New Hampshire, USA.
}

While respiratory failure in cystic fibrosis (CF) frequently associates with chronic infection by Pseudomonas aeruginosa, no single factor predicts the extent of lung damage in CF. To elucidate other causes, we studied the autoantibody profile in CF and rheumatoid arthritis (RA) patients, given the similar association of airway inflammation and autoimmunity in RA. Even though we observed that bactericidal permeability-increasing protein (BPI), carbamylated proteins, and citrullinated proteins all localized to the neutrophil extracellular traps (NETs), which are implicated in the development of autoimmunity, our study demonstrates striking autoantibody specificity in CF. Particularly, CF patients developed anti-BPI autoantibodies but hardly any anti-citrullinated protein autoantibodies (ACPA). In contrast, ACPA-positive RA patients exhibited no reactivity with BPI. Interestingly, anti-carbamylated protein autoantibodies (ACarPA) were found in both cohorts but did not cross-react with BPI. Contrary to ACPA and ACarPA, anti-BPI autoantibodies recognized the BPI C-terminus in the absence of posttranslational modifications. In fact, we discovered that $P$. aeruginosa-mediated NET formation results in BPI cleavage by $P$. aeruginosa elastase, which suggests a novel mechanism in the development of autoimmunity to BPI. In accordance with this model, autoantibodies associated with presence of $P$. aeruginosa on sputum culture. Finally, our results provide a role for autoimmunity in CF disease severity, as autoantibody levels associate with diminished lung function.

Conflict of interest: The authors have declared that no conflict of interest exists.

Submitted: June 8, 2016 Accepted: September 15, 2016 Published: October 20, 2016

Reference information: JCI Insight. 2016;1(17):e88912. doi:10.1172/jci.insight.88912.

\section{Introduction}

Cystic fibrosis (CF) is the most common lethal genetic disease in individuals of European descent $(1,2)$. Mutations in the CF transmembrane conductance regulator (CFTR) gene disrupt CFTR-controlled secretion of bicarbonate and chloride across airway epithelial cells (3). This impairment in ion transport across the epithelium enhances mucus viscosity and interferes with mucociliary clearance, thereby creating an environment susceptible to bacterial infection $(4,5)$. As a result, $\mathrm{CF}$ patients have recurrent respiratory bacterial infections, frequently involving Pseudomonas aeruginosa, which is detected in up to $85 \%$ of patients (6-8). Inevitably, persistent bacterial infection in CF patients leads to an influx of neutrophils into the lung that results in a state of chronic inflammation (9-12). Despite this characteristic immunopathology, CF patients demonstrate great clinical variability in their lung manifestations, which cannot be predicted by the nature of the CFTR mutation or the extent of bacterial infection (11-13).

Autoantibodies directed against bactericidal permeability-increasing protein (BPI) are found in $\mathrm{CF}$ patients and correlate with diminished lung function $(14,15)$. BPI $(\sim 55 \mathrm{kDa})$ is an antimicrobial peptide stored in azurophilic granules of neutrophils that is required for efficient clearance of gram-negative bacteria $(16,17)$. However, there is little understanding of the etiopathogenic role of this autoimmunity in CF. Studies of chronic inflammatory diseases, such as rheumatoid arthritis (RA), lupus, or granulomatosis with polyangiitis, have demonstrated a strong link between neutrophil-mediated inflammation and autoimmunity (18-20). Moreover, in RA, this inflammation is thought to initiate in the lung as a result of environmental factors, e.g., microbes or smoking (21). In particular, NETosis, a mechanism by which neutrophils extrude their DNA and protein contents to form neutrophil extracellular traps (NETs), is thought to lead to the breaking of tolerance to citrullinated and carbamylated proteins in RA patients (22). In this model, 

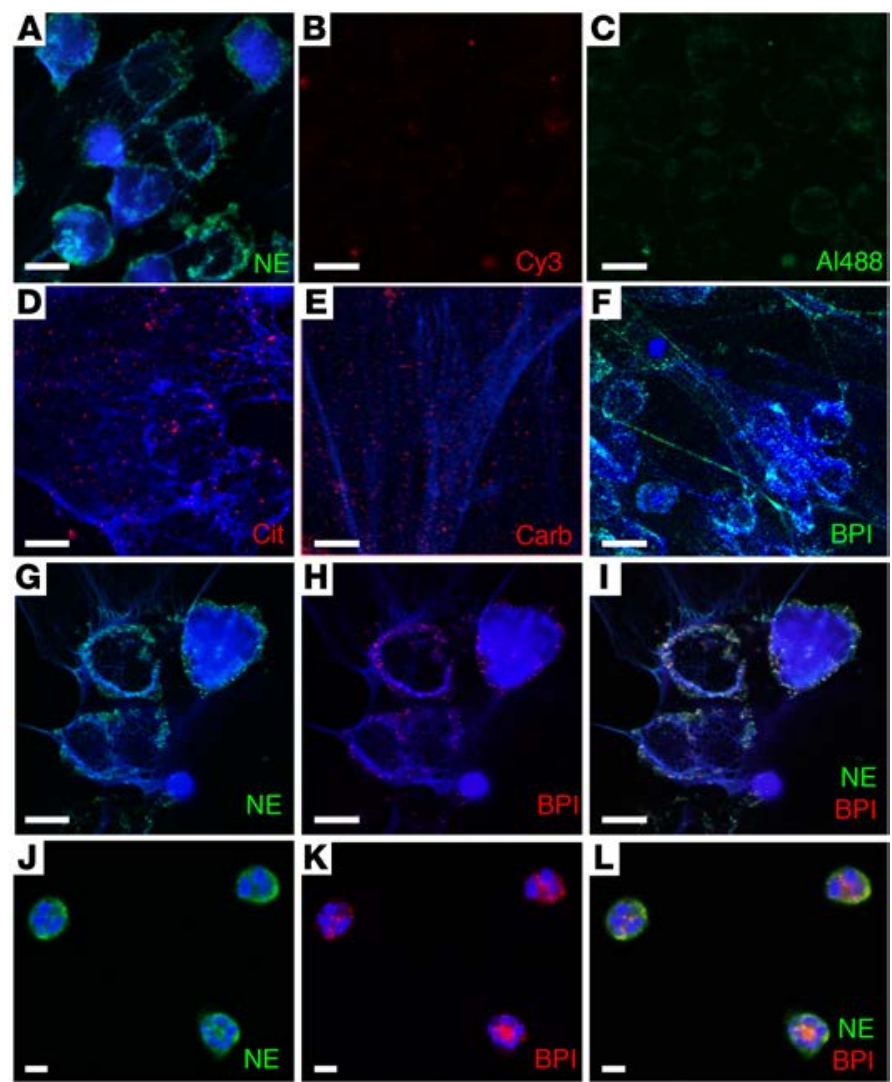

Figure 1. BPI and carbamylated proteins are localized on neutrophil extracellular traps. (A) PMA treatment ( $600 \mathrm{nM}, 2$ hours) of healthy neutrophils induced formation of neutrophil extracellular traps (NETs), characterized by extrusion of DNA (DAPI, blue) and neutrophil elastase (Al488, green). (B and C) Cy3 and Al488 alone were used to determine the level of nonspecific staining. Immunocytochemistry staining demonstrated that (D) citrullinated proteins (Cy3, red), (E) carbamylated proteins (Cy3, red), and (F) BPI-Al488 localize to the DNA strands of the NETs. (G-I) Neutrophil elastase (AI488) and BPI-Cy3 colocalize in the NETs. (J-L) Neutrophil elastase (AI488) and BPI-Cy3 colocalize in untreated PMNs. Scale bar: $10 \mu \mathrm{m}$. NE, neutrophil elastase; BPI, bactericidal permeabilityincreasing protein; Cit, citrullinated proteins; Carb, carbamylated proteins.

neutrophil enzymes that localize to NETs induce posttranslational modifications, such as citrullination and carbamylation, thus creating neoantigens that lead to anti-citrullinated protein autoantibodies (ACPA) and anti-carbamylated protein autoantibodies (ACarPA) (22-26). Given the abundance of nucleic acids in NETs, the induction of autoantibodies by NETosis is likely to be facilitated by TLR 7/9-mediated B cell activation (27). While formation of NETs in the CF lung has been appreciated as both an antibacterial defense mechanism and a contributor to proteaseinduced lung damage $(10,28-30)$, the role of NETosis in CF autoimmunity has not been studied.

Even though pulmonary insufficiency remains the leading cause of morbidity and mortality in CF, the causes and manifestations of chronic airway inflammation appear to differ between CF patients (31-35). This interpatient variability could result from unique underlying CFTR defects, differences in microbial infection, the associated immune responses, environmental influences, and disease-modifying genes $(36,37)$. Both the innate and adaptive immune systems shape the inflammatory environment of the CF lung and contribute to a complex and variable immunopathology that is not completely understood (38-40). In this article, we assess the nature of adaptive immunity in CF by comparing the autoantibody profile seen in adult CF and RA patients. As part of this study, we demonstrate the specificity of the anti-BPI immune response in CF and characterize its association with other known autoantibodies, bacterial infection, and lung function. Moreover, we propose the mechanism that leads to the breaking of tolerance to BPI in CF.

\section{Results}

BPI and other autoantibody targets in inflammatory diseases localize to the NETs. Following PMA-induced NET formation, we observed the expression of neutrophil elastase as well as citrullinated and carbamylated proteins on the decondensed DNA strands (Figure 1, A, D, and E). Moreover, BPI was found on the neutrophil membranes as well as on the NET DNA strands (Figure 1F). BPI frequently colocalized with neutrophil elastase, perhaps unsurprising given the dual release of these proteins from azurophilic granules (Figure 1, G-I, and J-L). Thus, BPI, like other autoantigens, is expressed in the context of extruded DNA in the NETs.

Autoantibody profile and specificity in CF patients. Given the presence of multiple autoantigens on NETs, we examined the possibility of overlapping autoantibody reactivity in CF $(n=38)$ and RA $(n=50)$. Autoantibodies targeting neutrophil-purified BPI (nBPI) were detected in $42 \%$ of CF serum samples, while none was detected in RA serum samples (Figure 2A and Table 1). ACPA-IgG and IgM rheumatoid factor were detected in less than $8 \%$ of $38 \mathrm{CF}$ serum samples in our cohort and at very low levels compared with RA patients (Figure 2, B and C, and Table 1). These findings suggest that autoimmunity to BPI and citrullinated proteins develops via disease-specific mechanisms. In contrast, ACarPA were detected in $28 \%$ of RA serum samples and in $40 \%$ of CF serum samples (Figure 2D and Table 1). Thus, the presence of frequent ACarPA reactivity in CF and RA patients supports the notion of a shared pathway in the breaking of tolerance to this particular neoantigen. 
A

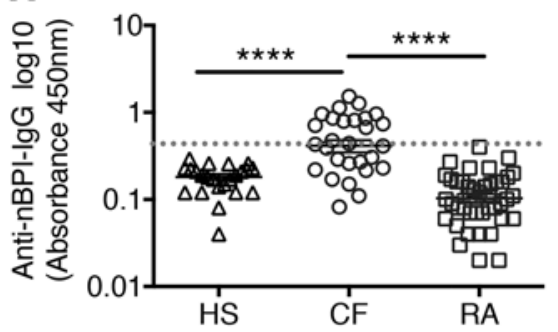

\section{C}

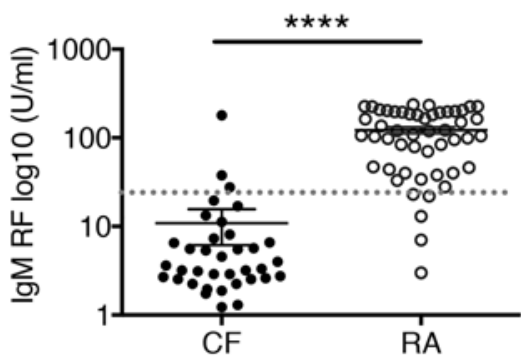

B

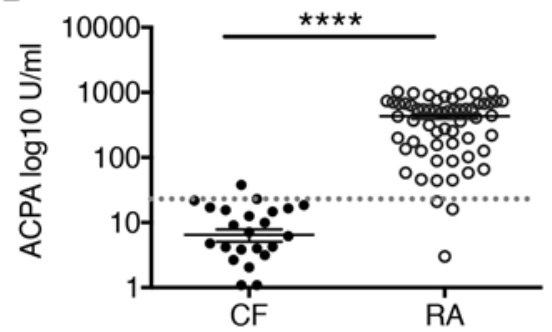

D

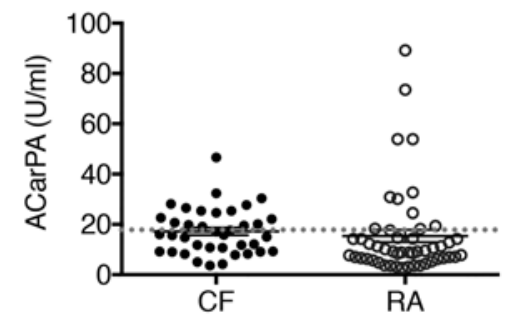

Figure 2. Autoantibody profile and specificity in CF patients. (A) Anti-nBPI IgC levels are higher in cystic fibrosis (CF) patients compared with those in healthy sera (HS) and rheumatoid arthritis (RA) patients by 1-way ANOVA with Bonferroni post-hoc (HS [ $n=23]$, mean $=0.15 ; \mathrm{CF}[n=38]$, mean $=0.41 ; \mathrm{RA}[n=50]$, mean $=0.10$ ); dotted line represents positive cutoff determined as mean +2 SD of HS cohort. (B) ACPA IgC levels are higher in RA patients compared with CF patients by $t$ test (CF $[n=$ 38], geometric mean $[\mathrm{Cl}=95 \%]=6.47 \mathrm{U} / \mathrm{ml} ; \mathrm{RA}$ $[n=50]$, geometric mean $[\mathrm{Cl}=95 \%]=430.5 \mathrm{U} /$ $\mathrm{ml})$; samples with ACPA levels $>20 \mathrm{U} / \mathrm{ml}$ were considered to be ACPA positive. (C) IgM rheumatoid factor (RF) levels are higher in RA patients compared with CF patients by $t$ test (CF $[n=38]$, geometric mean $[\mathrm{Cl}=95 \%]=10.9 \mathrm{U} / \mathrm{ml} ; \mathrm{RA}[n$ = 50], geometric mean $[\mathrm{Cl}=95 \%]=122 \mathrm{U} / \mathrm{ml}$ ); samples from patients with IgM RF concentrations $>25 \mathrm{U} / \mathrm{ml}$ were considered to be IgM RF positive. (D) ACarPA IgG are present at comparable levels in CF and RA patients by $t$ test (CF $[n=38]$, geometric mean $[\mathrm{Cl}=95 \%]=17.2 \mathrm{U} /$ $\mathrm{ml}$; RA $[n=50]$, geometric mean $[\mathrm{Cl}=95 \%]=$ $15.4 \mathrm{U} / \mathrm{ml}$ ); samples with ACarPA concentrations $>18 \mathrm{U} / \mathrm{ml}$ were considered to be ACarPA positive (measured by Inova Diagnostics). (A-D) Error bars represent mean $\pm \mathrm{SEM}$; ${ }^{* * * *} P<0.0001$. (E) Reactivity of $C F$ sera to denatured fibrinogen (Fib), carbamylated fibrinogen (carbam. Fib.), and $\mathrm{nBPI}(1 \mu \mathrm{g})$ was evaluated by immunoblot. Representative CF sera bind to $\mathrm{nBPI}$ and carbamylated fibrinogen with specificity. ACPA, anticitrullinated protein autoantibodies; ACarPA, anti-carbamylated protein autoantibodies; nBPI, neutrophil-purified BPI.

Therefore, the relationship between anti-nBPI autoantibodies and ACarPA was examined in CF sera. Surprisingly, no correlation between anti-nBPI autoantibodies and ACarPA was found in CF patients by either immunoblotting (Figure 2E) or ELISA (Supplemental Figure 1; supplemental material available online with this article; doi:10.1172/jci.insight.88912DS1). Selected CF sera exhibited distinct patterns of reactivity with either nBPI or carbamylated fibrinogen by immunoblotting (Figure 2E). Moreover, several $\mathrm{CF}$ serum samples reacted with both fibrinogen and carbamylated fibrinogen but not with nBPI. Reactivity of CF sera to carbamylated fibrinogen parallels previously observed autoreactivity in RA patients (41). Thus, autoantibodies in CF sera react with specificity with the denatured forms of nBPI, fibrinogen, and carbamylated fibrinogen. Despite being coexpressed in some CF serum samples, anti-nBPI and ACarPA lack cross-reactivity and appear to develop independently.

Autoantibody responses to BPI are directed at its $C$-terminus and do not require posttranslational modification. $\mathrm{CF}$ patient sera bound BPI in azurophilic granules of neutrophils in situ, thereby suggesting that anti-BPI autoantibodies do not detect posttranslationally modified neoantigens induced by NET formation. This was confirmed in several ways. First, no posttranslational modifications (carbamylation, citrullination, or isoaspartylation) of BPI were detected by proteomic analysis and isoAsp vapor assay (Supplemental Tables 1 and 2). Consistent with this finding, we did not detect colocalization between BPI and proteins containing either carbamylated or citrullinated neoepitopes by immunocytochemistry in PMA- or P. aeruginosa-induced NETs (Supplemental Figure 2). Second, using recombinant protein corresponding to the C-terminus of BPI (CTBPI, aa 227-487), we demonstrated a high level of correlation between IgG reactivity toward this protein fragment and that toward nBPI by ELISA and immunoblotting $(r=0.69, P<0.0001)$ (Figure 3). Unsurprisingly, none of the RA sera reacted with CTBPI recombinant protein (data not shown). Thus, the reactivity of anti-BPI autoantibodies maps to CTBPI and derives from the primary amino acid sequence in the absence of any posttranslational modifications in the bacterially derived recombinant protein. 
Table 1. Autoantibodies in chronic inflammatory diseases

\begin{tabular}{lcc}
\hline & \multicolumn{2}{c}{$\%$ Positive } \\
& CF sera $(\boldsymbol{n}=\mathbf{3 8})$ & RA sera $(\boldsymbol{n}=\mathbf{5 0})$ \\
ACPA $^{\mathrm{A}}$ & $5.3 \%(2 / 38)$ & $94 \%(47 / 50)$ \\
IgM RF $^{\mathrm{B}}$ & $7.8 \%(3 / 38)$ & $92 \%(46 / 50)$ \\
Anti-BPI IgG $^{\mathrm{C}}$ & $42 \%(16 / 38)$ & $0 \%(0 / 50)$ \\
ACarPA $^{\mathrm{D}}$ & $40 \%(15 / 38)$ & $28 \%(14 / 50)$
\end{tabular}

${ }^{A} A C P A$ positive, ACPA concentration $>20 \mathrm{U} / \mathrm{ml}$. ${ }^{B}$ IgM RF positive, IgM RF concentration $>25 \mathrm{U} / \mathrm{ml}$. 'Anti-BPI IgG positive, anti-BPI IgG concentration $>0.4 \mathrm{OD}_{450} \cdot{ }^{\circ} \mathrm{AC}$ CarPA positive, ACarPA concentration $>18 \mathrm{U} / \mathrm{ml}$. ACarPA, anti-carbamylated protein autoantibodies; ACPA, anti-citrullinated protein autoantibodies; CF, cystic fibrosis; IgM RF, IgM rheumatoid factor; RA, rheumatoid arthritis.
Differential BPI cleavage occurs in a $P$. aeruginosa strain-dependent manner. The C-terminal domain of BPI mediates the interaction of bacterial proteins with DCs (42). Based on our finding that anti-BPI IgG reactivity is specific to the CTBPI, we postulated that BPI cleavage might facilitate its delivery to the DCs in the context of NETosis and so lead to the breaking of tolerance. Moreover, the association of BPI autoantibodies and CF prompted us to examine the potential role of $P$. aeruginosa in BPI cleavage. Following incubation of neutrophils with $P$. aeruginosa strain PA14, immunoblotting with a mouse monoclonal anti-human BPI antibody specific for aa 227-254 revealed the appearance of multiple proteins smaller than $55 \mathrm{kDa}$, including an appropriately $30-\mathrm{kDa}$ fragment that corresponds to CTBPI (Figure 4A). This effect appeared specific for PA14, as it was not seen with NET induction by PMA or glucose oxidase treatment (Figure 4A). Similar, dose-dependent, effects were seen with different $P$. aeruginosa MOI (PA14 or PAO1) (Figure 4B). Because an elastase cleavage site separates the $\mathrm{N}$-terminus and the C-terminus domains of BPI, we evaluated the role of $P$. aeruginosa elastase in BPI cleavage (43). Immunoblotting demonstrated that treatment of neutrophils with a $P$. aeruginosa elastase-specific knockout bacterial strain ( $\triangle$ lasB PAO1) did not induce the appearance of the appropriately $30-\mathrm{kDa}$ BPI fragment, in contrast to the wild-type PAO1 treatment (Figure 4C) (44). Similar results were obtained following treatment with $\triangle$ las $R$ PAO1 and $\triangle$ lasR PA14, additional elastase-deficient $P$. aeruginosa strains (Figure $4 C)$. Variable BPI cleavage was observed in the untreated PMNs, suggesting an effect of neutrophil elastase (NT, Figure 4C). In the presence of elastase-deficient $P$. aeruginosa, this effect of neutrophil elastase appears attenuated, as BPI cleavage was only seen following treatment with elastase expressing $P$. aeruginosa. Therefore, our findings suggest that BPI cleavage in $P$. aeruginosa-induced NETs occurs in a $P$. aeruginosa elastase-dependent manner. The potential contributions of neutrophil elastase to BPI cleavage require further examination. In addition, CTBPI protein fragments were detected in bronchoalveolar lavage samples of patients with anti-CTBPI autoreactivity (Figure 4D).

Clinical correlations of autoimmunity in $C F$. The relationship between anti-BPI reactivity and clinical and serologic features in our adult $\mathrm{CF}$ cohort was examined (Table 2 and Supplemental Table 3). We observed a significant negative correlation between anti-CTBPI IgG titers and forced expiratory volume in 1 second (FEV1 percent predicted, $r=$ $-0.66, P<0.0001$ ) (Figure 5A), which demonstrated that anti-CTBPI IgG autoantibodies trend with

Figure 3. Anti-BPI autoantibodies in CF target the C-terminus of BPI. (A) Crystal structure of BPI protein (RCSB Protein Data Bank, accession number P17213.4); diagram shows N-terminus (NT-BPI, aa 10-240) and C-terminus (CTBPI, aa 260-430). (B) Reactivity of cystic fibrosis (CF) sera to the recombinant CTBPI correlates with reactivity to the intact $\mathrm{nBPI}$, as determined by Pearson correlation analysis ( $r=0.690, P<0.0001, n=38$ ). (C) Recombinant CTBPI and nBPI $(1 \mu \mathrm{g})$ were detected by immunoblot using monoclonal anti-BPI antibody specific for the epitope mapping to aa 227-254. (D) Representative immunoblots confirm reactivity of CF sera to the total BPI (55 kDa, left lane) and to recombinant CTBPI protein ( $30 \mathrm{kDa}$, right lane). $\mathrm{nBPI}=$ neutrophil-purified bactericidal permeability-increasing protein.

$\mathbf{A}$
B

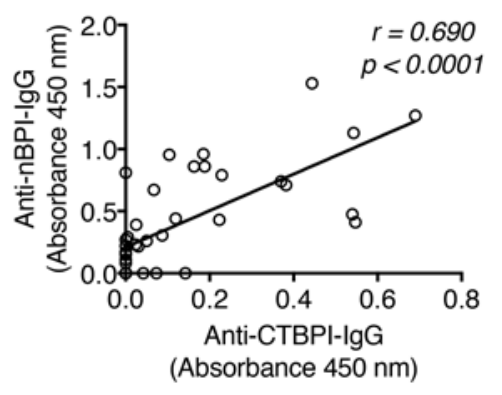

D

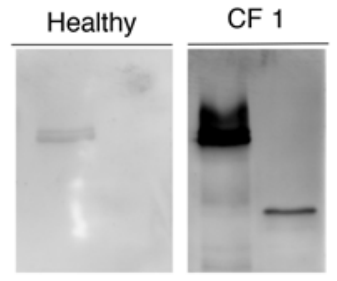

c

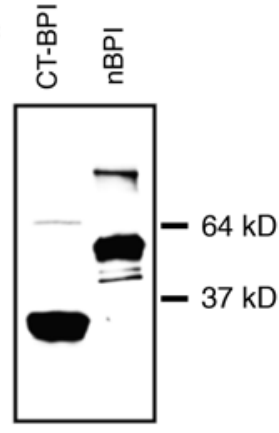

CF 2

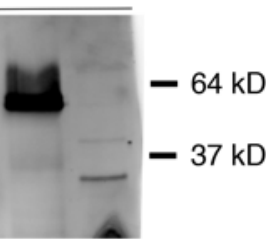


Table 2. Clinical characteristics of CF patient cohort

\begin{tabular}{|c|c|c|c|c|}
\hline Patient population & $n$ & Percentage (\%) & $\begin{array}{l}\text { FEV1 percent predicted } \\
(\text { Mean } \pm \text { SD) }\end{array}$ & $\begin{array}{c}\text { Age (yr) } \\
(\text { Mean } \pm \text { SD) }\end{array}$ \\
\hline Total & 38 & & & \\
\hline Males & 25 & $66 \%$ & $68 \pm 27$ & $32 \pm 11$ \\
\hline Females & 13 & $34 \%$ & $59 \pm 21$ & $30 \pm 6$ \\
\hline \multicolumn{5}{|l|}{ Age (yr) } \\
\hline Mean & 30.9 & - & - & - \\
\hline Range & $18-63$ & - & - & - \\
\hline \multicolumn{5}{|c|}{ CFTR mutation class } \\
\hline Class I & 5 & $13.2 \%$ & $44 \pm 27$ & $41 \pm 17$ \\
\hline Class II & 24 & $63 \%$ & $62 \pm 27$ & $28 \pm 8$ \\
\hline Class III & 2 & $5.3 \%$ & $60 \pm 5$ & $34 \pm 1$ \\
\hline Class IV & 1 & $2.6 \%$ & 26 & 19 \\
\hline Class V & 5 & $13.2 \%$ & $50 \pm 28$ & $37 \pm 6$ \\
\hline Unclassified & 1 & $2.6 \%$ & 23 & 24 \\
\hline \multicolumn{5}{|c|}{ FEV1 percent predicted } \\
\hline$>80 \%$ & 9 & $24 \%$ & - & $26 \pm 5$ \\
\hline $50-80 \%$ & 12 & $32 \%$ & - & $28 \pm 5$ \\
\hline$<50 \%$ & 17 & $45 \%$ & - & $35 \pm 13$ \\
\hline \multicolumn{5}{|c|}{ P. aeruginosa infection } \\
\hline Positive & 29 & $76 \%$ & $52 \pm 26$ & $32 \pm 11$ \\
\hline Negative & 9 & $24 \%$ & $69 \pm 26$ & $28 \pm 8$ \\
\hline
\end{tabular}

Class I: F508del/G542X, F508del/621+1G>T, F508del/711+1G>T, F508del/3659delC, UNKN/1717-1G>T. Class II: F508del/F508 del. Class III: F508del/G551D. Class IV: F508del/H1085R. Class V: F508del/A455E. Unclassified: F508del/EX6b_10dup. deteriorating lung function in adult CF patients. Similar correlation was found with anti-nBPI autoantibody titers $(P=0.003, r=-0.468$, data not shown) as well as with FEV1 Z score values ( $n=19, P=0.071, r=$ -0.40 , data not shown). Significantly higher autoantibody levels were also detected in patients who experienced more than two pulmonary exacerbations per year $(P=0.011$, data not shown). Presence of these autoantibodies in the bronchoalveolar lavage fluid of CF patients suggests that the autoimmune response might arise locally in the CF patient airway (Figure 5B). Moreover, incidence of antiCTBPI IgG strongly associated with $P$. aeruginosa-positive sputum culture as well as with infection with the mucoid form of $P$. aeruginosa, which is associated with increased morbidity and mortality in CF (Figure 5, C and D) (45). Furthermore, patients that were homozygous for the F508 deletion mutation (F508del) in CFTR had significantly lower levels of serum anti-nBPI IgG than $\mathrm{CF}$

patients with F508del heterozygosity or a different CFTR mutation (Figure 5E). As with the associations of anti-BPI autoimmunity with lower FEV1 percent predicted scores, we observed a trend toward better pulmonary function (i.e., higher FEV1 percent predicted) in patients with F508 deletion homozygosity (62\% $\pm 27 \%$ ) than in patients with other mutation classes $(47 \% \pm 24 \%)(P=0.09)$. (Table 2 and data not shown). Similarly, F508 deletion homozygous patients had a lower prevalence of $P$. aeruginosa infection $(70.8 \%)$ than patients with a different CFTR mutation class (85.7\%) (data not shown).

Our model proposes that the breaking of tolerance to BPI is facilitated by $P$. aeruginosa. Interestingly, BPI autoreactivity was associated with increased levels of antibodies toward the CFTR inhibitory factor (Cif) (Figure 5F), a P. aeruginosa virulence factor. No relationship was seen with anti-PA14 antibody responses, despite higher levels of anti-PA14 IgG in CF sera (Supplemental Figure 3, A and B). Similarly, we observed no relationship between anti-BPI autoantibody levels and total serum IgG titers, indicating that autoreactivity to BPI is not a consequence of hypergammaglobulinemia or diffuse dysregulation of the humoral immune response (Supplemental Figure 3C). As previously reported, higher levels of total IgG were seen in CF sera relative to the healthy controls (Supplemental Figure 3D) (46). A significant but less striking relationship was also seen between ACarPA and the clinical parameters of disease progression, diminished lung function (FEV1 percent predicted, $r=-0.278, P=0.046)$ and $P$. aeruginosa infection $(P=$ 0.01) (Supplemental Figure 4, A-C). Intriguingly, the associations of ACarPA in CF patients were detected irrespective of anti-Cif IgG presence (Supplemental Figure 4D).

\section{Discussion}

In this article, we report that BPI and carbamylated proteins are associated with NETs, as has been previously observed with citrullinated proteins (47). Second, we demonstrated that autoantibodies to BPI and carbamylated, but not citrullinated, proteins occur in CF patients. However, anti-BPI autoantibodies and ACarPA do not exhibit patterns of coexpression or cross-reactivity in CF patient sera. Third, we showed that antineutrophil BPI autoreactivity maps to the CTBPI and does not appear to require posttranslational modification. This is the first study to our knowledge to show anti-BPI IgG reactiv- 
A

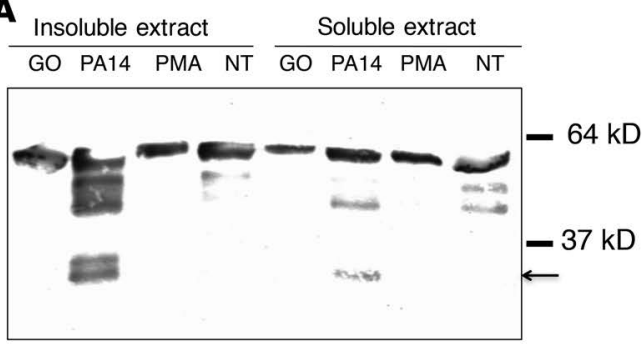

C

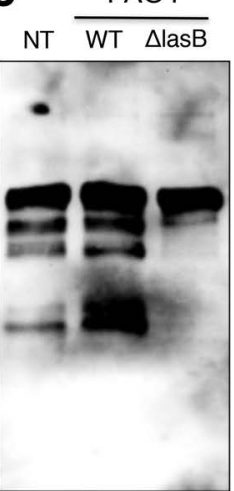

$\mathrm{PAO} 1$

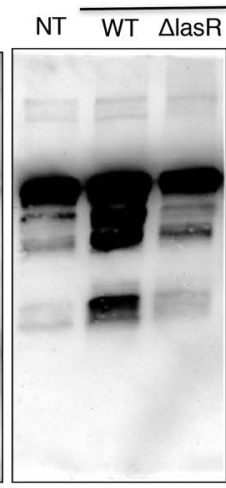

B
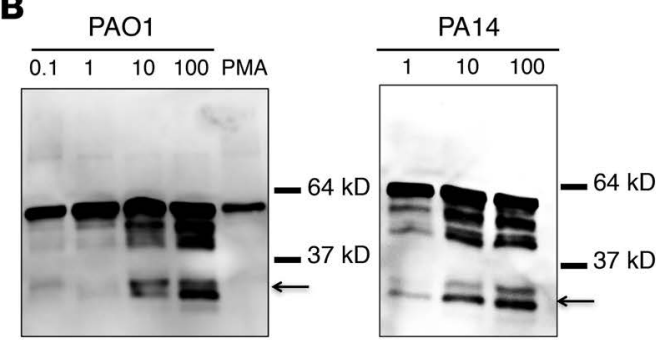

D
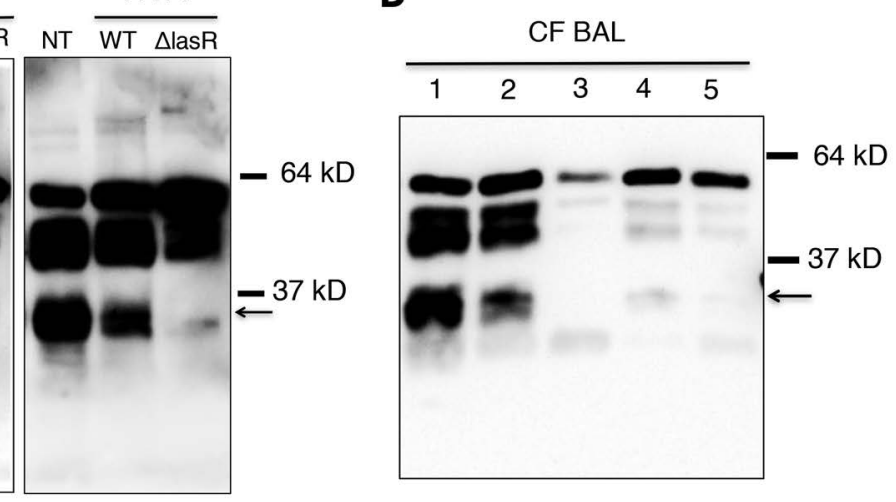

Figure 4. BPI is cleaved in a $\boldsymbol{P}$. aeruginosa strain-dependent manner. (A) Healthy neutrophils were treated with NET-inducing agents, PMA (20 nM), P. aeruginosa PA14 (100 MOI), or glucose oxidase $(\mathrm{GO}, 2 \mathrm{U} / \mathrm{ml})$, or left untreated (NT) for 1 hour. Total BPI protein $(55 \mathrm{kDa}$ ) and smaller protein fragments were detected in $20 \mu \mathrm{g}$ of insoluble and soluble protein extracts via immunoblot with a mouse anti-human BPI antibody directed at aa 227-254 epitope (i.e., BPI C-terminus hinge region). (B) Total BPI and protein fragments were detected by immunoblot in soluble protein extracts $(10 \mu \mathrm{g})$ from neutrophils treated with PMA or increasing MOIs of $P$. aeruginosa strains PA01 $(0.1,1$, 10 , and $100 \mathrm{MOI})$ and PA14 (1, 10, and $100 \mathrm{MOI})$ with the antibody used in A. (C) The extent of BPI cleavage detected in soluble protein extracts $(10 \mu \mathrm{g})$ from neutrophils following incubation with $P$. aeruginosa strains PA01 and PA14, wild type strains (WT), or elastase deficient strains ( $\triangle$ lasB or $\Delta$ lasR) (10 MOI) for 1 hour. (D) Total BPI and protein fragments were detected by immunoblot in BAL samples ( $20 \mu$ g protein/sample) with the antibody used in $\mathbf{A}$. The immunoblots in A-C are representative images of $n=3$ experiments. NET, neutrophil extracellular trap; BPI, bactericidal permeability-increasing protein; BAL, bronchoalveolar lavage.

ity to an isolated recombinant BPI C-terminus. Fourth, we found that neutrophil BPI is cleaved in a $P$. aeruginosa elastase-dependent manner, which provides a possible model for the breaking of tolerance to BPI. Finally, in the first-ever analysis of autoimmunity in an adult US CF cohort, we demonstrated that autoreactivity to BPI reflects diminished airway function, which arises in the context of P. aeruginosa infection and the CFTR genotype.

Since the breaking of tolerance in RA involves lung injury themes common to CF (airway irritants, bronchial inflammation findings on chest $\mathrm{CT}$, and autoantigen-decorated NETs), we initially hypothesized that autoimmunity in CF might parallel the autoantibody profile in RA (48-52). However, anti-BPI IgG and ACPA segregated with CF and RA patients, respectively. In a panel of RA sera enriched for ACPA and ACarPA seropositivity, we observed a lower frequency of anti-BPI autoantibodies in RA patients than previously reported in a serologically undefined RA patient population (53). In RA, there is a strong association of ACarPA with ACPA (24). In contrast, despite seeing ACarPA in CF patients, we observed a poor correlation between ACarPA and anti-BPI autoantibodies. Moreover, neither autoantibody associated with hypergammaglobulinemia, suggesting that autoantibody development was not due to a nonspecific increase in B cell growth and differentiation. Additionally, in contrast to RA, we showed that autoimmunity to BPI in CF does not appear to result from posttranslational modifications. Since persistent bacterial infections are one of the major triggers of chronic inflammation in the $\mathrm{CF}$ airway, we explored the relationship between $P$. aeruginosa infection, which is present in up to $85 \%$ of CF patients, and the development of autoreactivity to BPI.

This is the first study to our knowledge to shed light onto the potential mechanisms involved in the breaking of tolerance to BPI. We demonstrate that $P$. aeruginosa elastase may be the culprit in generating 

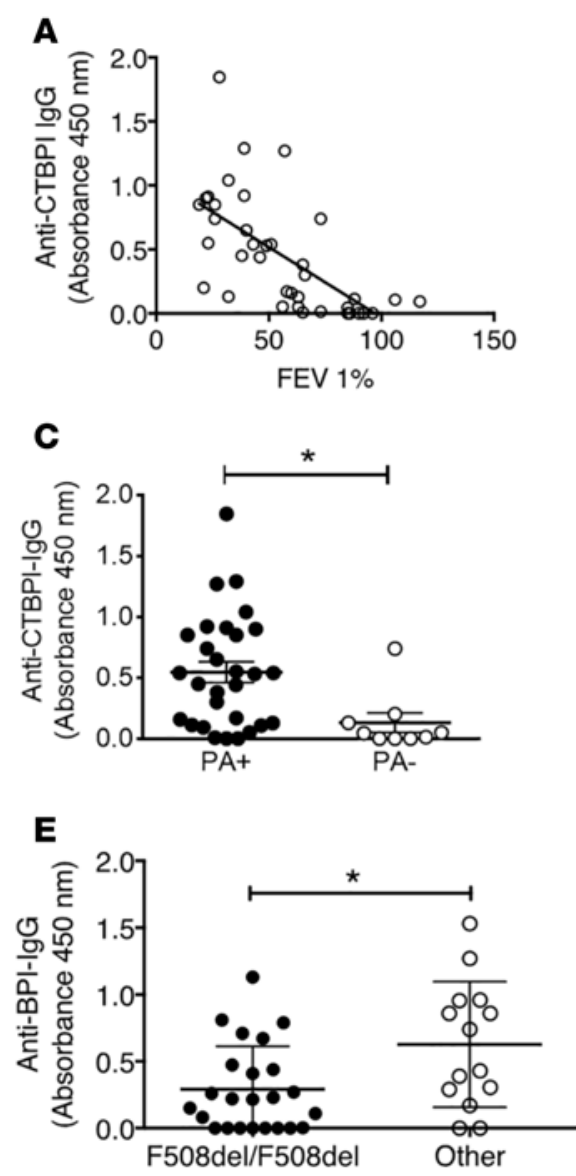

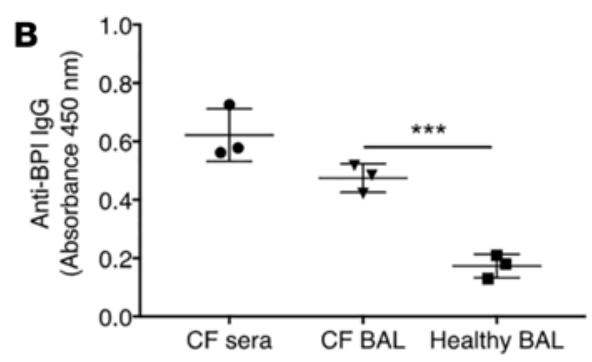

D

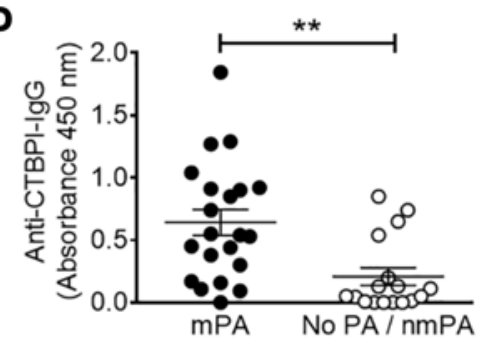

$\mathbf{F}$

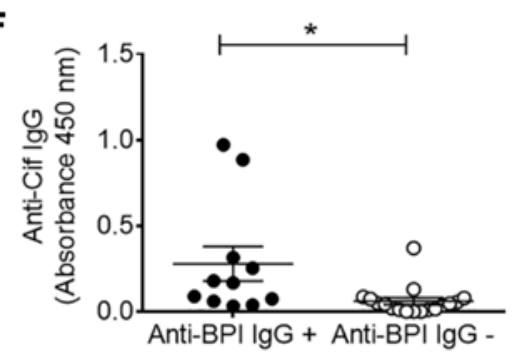

Figure 5. Anti-BPI IgG correlates with FEV1 and anti-Cif immune response to $P$. aeruginosa in a US adult CF cohort. (A) Anti-CTBPI IgC levels determined by ELISA negatively correlate with pulmonary function in cystic fibrosis (CF) patients (FEV1 percent predicted), as determined by Pearson correlation analysis $(n=38$, $r=-0.664, P<0.0001)$. $P$. aeruginosa infection status of patients is denoted: $P$. aeruginosa $a^{+}$ (white circle) and $P$. aeruginosa- (black circle). (B) Anti-BPI IgC titers were measured in representative CF bronchoalveolar lavage (BAL) samples that previously demonstrated serum anti-BPI reactivity by ELISA (ANOVA, $n=3$ ), ${ }^{* *} P<0.001$. (C) Higher anti-CTBPI IgC levels associate with positive $P$. aeruginosa sputum culture $\left(\mathrm{PA}^{+}\right)$ in CF patients ( $n=38)$, (D) particularly with presence of the mucoid $P$. aeruginosa strains (mPA) $(n=38)$. (E) Anti-BPI IgC titers are higher in CF patients that do not carry the F508del homozygous CFTR gene mutation $(n=38)$. (F) Anti-Cif $(10 \mu \mathrm{g} / \mathrm{ml})$ IgG levels are higher in CF patients with anti-BPI autoantibodies (Anti-BPI $\mathrm{IgG}^{+}$) compared with patients without reactivity to BPI (Anti-BPI IgG-) $(n=28)$. (C-F) Significance between two groups was determined by Student's $t$ test with Welch's correction; ${ }^{*} P<$ $0.05,{ }^{* *} P<0.01$, ${ }^{* * *} P<0.001$; error bars represent mean $\pm \mathrm{SEM}$. FEV $1 \%$, forced expiratory volume in 1 second percent predicted; Cif, CFTR inhibitory factor; BPI, bactericidal permeabilityincreasing protein.

neoepitopes, via BPI cleavage. Based on the studies using the recombinant BPI, the target epitope is likely located in the protein C-terminus, i.e., an appropriately $30-\mathrm{kDa}$ protein fragment detected following neutrophil treatment with wild-type $P$. aeruginosa strains. Our findings support the model in which BPI protein fragments are taken up by the airway antigen-presenting cells (e.g., DCs and monocytes), which leads to the activation of the adaptive immune response and, consequently, to the triggering of autoimmunity (14). In addition to the mechanistic relationship between $P$. aeruginosa elastase and BPI protein integrity, we observed a strong association of BPI autoreactivity with positive $P$. aeruginosa sputum culture, particularly with the more pathogenic mucoid strains. Moreover, presence of autoimmunity to BPI was concurrent with a humoral response directed at Cif (anti-Cif IgG), a virulence protein secreted by $P$. aeruginosa to inhibit CFTR activity (54-56). As expected, these associations were not apparent with ACarPA, which suggests that autoreactivity to BPI occurs specifically in the context of $P$. aeruginosa infection in CF. Adding to the complexity was the observation that high titers of anti-BPI autoantibodies associated with the absence of homozygous F508del CFTR mutation. These findings are interesting, as no direct association between the $C F T R$ mutation type and the extent of airway disease has been discovered thus far (57). Moreover, little is known about the role different CFTR mutation classes play in the intrinsic immune functions and responses in CF patients, particularly in the $P$. aeruginosa-infected lung (58). Our findings suggest that the CFTR mutation and particular $P$. aeruginosa strains may interact in the breaking of tolerance to BPI. However, we acknowledge that HLA genotyping might provide an additional insight into the susceptibility of CF patients to develop anti-BPI and/or ACarPA reactivity. In RA, ACPA formation appears highly associated with HLA-DRB1 haplotype (59). In contrast, ACarPA do not appear to share this association in RA (60). It is possible that as-yet undefined HLA haplotypes are associated with anti-BPI and ACarPA responses $(61,62)$.

The variability in the extent of airway compromise and damage in CF patients remains unexplained. High titers of anti-BPI autoantibodies associated with worse lung function, sputum culture positivity for the mucoid strain of P. aeruginosa, and the absence of homozygous F508del CFTR mutation. Lack of a 
functional BPI has been associated with decreased clearance of the mucoid strain of $P$. aeruginosa, which leads to biofilm formation and obstructed respiratory function (16). Furthermore, anti-BPI autoantibodies have been shown to neutralize the bactericidal and opsonic activity of BPI $(63,64)$. Therefore, levels of anti-BPI autoantibodies might influence airway compromise by neutralizing the antibacterial role of BPI. Moreover, the presence of chronic infection supports our model of a P. aeruginosa-driven mechanism of the breaking of tolerance to BPI. The presence of these autoantibodies in the bronchoalveolar lavage fluid of $\mathrm{CF}$ patients is consistent with the hypothesis that local airway infection with elastase-expressing $P$. aerugino$s a$ drives the development of this autoimmunity. P. aeruginosa has previously been shown to inactivate host immune responses in the CF lung by proteolytic cleavage of antibacterial peptides and proteins involved in airway homeostasis $(65,66)$. However, our findings do not exclude a role for neutrophil elastase in BPI cleavage, which is an important contributor to lung damage in CF (67).

In contrast, we note that another autoantibody type, ACarPA, demonstrates a moderate but noteworthy correlation with diminished pulmonary function (lower FEV1) and infection with P. aeruginosa. In contrast to anti-BPI responses, the lack of association between ACarPa and anti-Cif IgG levels suggests that ACarPA arise independently of infection and are most likely a marker of neutrophil-driven bronchial inflammation reported in other chronic diseases $(24,68,69)$. Alternatively, it may be that ACarPA influence clinical phenotype by impairing the antibacterial activity on NETs. Some limitations of our study are noted. The cohort size and the confounding effect of patient age may have influenced the associations of anti-BPI autoantibodies and ACarPA with clinical parameters. In particular, a larger analysis, including pediatric CF patients, would provide a better understanding of the role CFTR mutation class plays in the development of autoimmunity. Such a study would also begin to address any potential confounding role that age might play in autoimmunity. In addition, examining the full repertoire of autoantibodies in CF patients would provide a more complete picture of the immune response, as anti-PR3 autoantibodies have been reported in pediatric CF patients (70).

Our study provides insight into the development of autoimmunity to BPI, as well as to carbamylated proteins, in CF. Even though recurring flares lead to irreversible lung damage, no correlation has been found between changes in the microbiome and disease exacerbation (71). This study suggests that the level of anti-BPI autoantibodies can serve as a marker of deteriorating lung function in a subset of CF patients. Further studies will be required to understand the possible contributions of these autoantibodies to disease progression. Moreover, this work reveals a likely mechanism that leads to the breaking of tolerance in an environment of chronic $P$. aeruginosa infection, which has previously been identified as one of the triggers of autoimmune disease $(72,73)$. Future epitope mapping will determine the immunogenic peptide of the BPI protein. These studies could also provide an insight into the development of autoimmunity to BPI in other inflammatory lung diseases, such as bronchiectasis and interstitial lung disease $(74,75)$. Due to the complexity of the inflammatory environment in the lung, future studies of anti-BPI autoimmunity development and function should also address the role of neutrophil elastase, particularly its ability to cleave and degrade immunoglobulins $(76,77)$.

\section{Methods}

Patient cohorts. Sera were collected from a cohort of 38 adult CF patients treated at Dartmouth-Hitchcock Medical Center (DHMC), which is located in Lebanon, New Hampshire, USA. The mean age of the CF patients was 30.9 years (range 18-63 years), and 25 patients were male, while 13 were female. Sputum cultures and patient lung function at the time of serum collection were assayed by clinical laboratories at DHMC. No patient has undergone lung or liver transplantation. RA patient sera (94\% seropositive for ACPA and IgM rheumatoid factor; $n=50$ ) were stored in the Rheumatic Disease Biomarker Biorepository at DHMC.

Antibody detection. The frequency of seropositivity of the RA and CF patient cohorts was measured by CCP3 and the QUANTA Lite RF IgM ELISA kit (Inova Diagnostics), while serum IgG levels were measured using the Human IgG ELISA kit (ICL Inc.). Cif (a gift of Dean Madden at Geisel School of Medicine at Dartmouth) and P. aeruginosa PA14 lysates were used to create ELISA assays for measurement of antibodies to these proteins in CF and normal human sera (1:100 dilution).

Anti-BPI autoreactivity was measured in patients with CF and RA, and in healthy subjects, by an in-house ELISA coated with nBPI (Athens Research and Technology), which was validated $\left(R^{2}=0.85\right)$ against the commercial anti-BPI ELISA assay (Immuno-Biological Laboratories Inc.). After being coated with human nBPI $(10 \mu \mathrm{g} / \mathrm{ml})$, 96-well ELISA plates were blocked with PBS+1\% BSA overnight. Sera, 
diluted 1:100 in the dilution buffer (PBS+1\%BSA), or undiluted bronchoalveolar lavage fluid were incubated for 1 hour at room temperature. Anti-human IgG $(\mathrm{H}+\mathrm{L}), \mathrm{F}(\mathrm{ab}) 2$ fragment, and peroxidase-labeled antibody (KPL Inc.) (1:50,000) were added, followed by R\&D Systems substrate and stop solution. Absorbance was read at $450 \mathrm{~nm}$ using ELISA reader (Epoch, BioTek). The presence of anti-BPI IgG in CF sera was verified by fluid-phase inhibition ELISA, where $50 \mu \mathrm{g}$ nBPI was used as inhibitory agent (30 minutes at room temperature), prior to the ELISA assay. A minimum of a $30 \%$ reduction in $\mathrm{OD}_{450 \mathrm{~nm}}$, relative to the serum control, was considered meaningful (Supplemental Figure 5). Autoantibodies to CTBPI were also measured by ELISA. The recombinant CTBPI was produced by TOPO Cloning technology (ThermoFisher Scientific). The primers used for the pET1D1/D-TOPO-6XHis tag vector are as follows: 5'CACCATGAACAGCCAGGTCTGCGAGA3' (upper) and 5'TTTATAGACAACGTC TGCACCG3' (lower). Nickel-coated ELISA plates (ThermoFisher Scientific) were used to bind the 6X-His tagged CTBPI (10 $\mu \mathrm{g} /$ $\mathrm{ml}$ ), and ELISA was performed as described for nBPI. Anti-carbamylated protein IgG titers (ACarPA) in sera were evaluated by commercial ACarPA ELISA assay (Inova Diagnostics Inc.) as well by a previously described in-house ACarPA ELISA (24). Positive cutoff for the BPI ELISA assays was defined as mean + 2 SDs of the healthy donor cohort.

Detection of autoantibody reactivity by immunoblot. Immunoblotting of nBPI, native fibrinogen (SigmaAldrich), and carbamylated fibrinogen $(1 \mu \mathrm{g})$ was performed after resolution by SDS-PAGE (12\% acrylamide gel) and transfer to a nitrocellulose membrane (Sigma-Aldrich). Following blocking in tris buffer saline with $0.05 \%$ tween-20 (TBS-T) $+3 \%$ BSA, the membranes were probed with patient serum $(1: 100$ in TBS-T $+1 \%$ BSA) for 1 hour at room temperature, washed, and incubated with goat anti-human peroxidase-labeled secondary antibody (1:400,000, in TBS-T + 1\% BSA). Enhanced chemiluminescent substrate SuperSignal West Femto (ThermoFisher Scientific) was used for protein detection via the Syngene G-Box system and software (Synoptics). A slightly modified protocol was utilized to subsequently detect reactivity of CF sera to nBPI and CTBPI via immunoblot: nitrocellulose membranes were blocked in TBS-T + $10 \%$ FCS overnight and probed with CF sera (1:250, in TBS-T + 3\% FCS) for 2 hours; goat anti-human peroxidase-labeled secondary antibody was used at 1:6,000 dilution; and the enhanced chemiluminescent substrate SuperSignal West Pico (ThermoFisher Scientific) was used for detection.

NET induction and immunocytochemistry. Induction and visualization of NETs was done as previously described (78). Following Ficoll discontinuous gradient separation, neutrophils were purified from the erythrocyte pellet by $5 \%$ dextran sedimentation. Neutrophils were seeded onto glass coverslips $\left(2 \times 10^{5}\right.$ cells), activated with $600 \mathrm{nM}$ PMA for 2 hours, and fixed in 4\% paraformaldehyde. Rabbit anti-human neutrophil elastase antibody (Abcam) and rabbit anti-BPI antibody (Sigma-Aldrich) were directly labeled with Alexa Fluor 488 and Alexa Fluor 568 protein labeling kits (ThermoFisher Scientific), respectively; 100 $\mu \mathrm{g} / \mathrm{ml}$ anti-neutrophil elastase-AI488 and $50 \mu \mathrm{g} / \mathrm{ml}$ anti-BPI-AI568 were used for immunocytochemistry. BPI was also detected using mouse anti-BPI antibody (1:200, Santa Cruz Biotechnology Inc.), followed by donkey anti-mouse Alexa Fluor 488 antibody (1:500, Jackson ImmunoResearch). Carbamylated and citrullinated proteins were detected using rabbit anti-carbamyl lysine antibody ( $\alpha \mathrm{CBL}$, Cell Biolabs Inc.) and rabbit anti-citrulline antibody (Abcam) (1:200), followed by donkey anti-rabbit Cy3 antibody (1:500, Jackson ImmunoResearch). Samples were mounted using ProLong Gold Antifade Mount with DAPI (ThermoFisher Scientific) and visualized with the laser point scanning confocal microscope (LSM 510 META, Zeiss; $\times 60$ ). Donkey anti-rabbit Cy3 and donkey anti-mouse A1488 secondary antibodies were used alone to determine non-specific staining (Figure $1, \mathrm{~B}$ and $\mathrm{C}$ ).

Detection of BPI posttranslational modifications. A $55-\mathrm{kDa}$ band corresponding to purified $\mathrm{nBPI}(10 \mu \mathrm{g}$, Athens Research and Technology) was resolved by a 12\% SDS-PAGE gel; the band was cut out and sent for proteomic analysis for citrullinated and carbamylated posttranslational modifications by tandem mass spectrometry at the Mass Spectrometry \& Proteomics Resource at the W.M. Keck Foundation Biotechnology Resource Laboratory (Yale School of Medicine). Isoaspartylation (isoAsp) in purified BPI protein was measured by isoAsp vapor assay as described previously (79). 50 pmole isoAsp containing $\delta$ sleep-inducing peptide (isoAsp-DSIP) was used as a positive control, and a reaction mix without isoAsp-DSIP was applied as a negative control. The presence of posttranslational modifications of BPI was also investigated using immunocytochemistry, as described above.

Detection of BPI cleavage by P. aeruginosa. P. aeruginosa PA14 and PAO1 strains were provided by Brent Berwin at the Geisel School of Medicine at Dartmouth. The mechanism of BPI cleavage was investigated using PAO1 $\triangle$ lasR and PA14 AlasR bacterial strains provided by Deborah Hogan at the Geisel School of 
Medicine at Dartmouth as well as using the PAO1 $\triangle$ lasB strain, a gift of Dao Nguyen laboratory at McGill University, Montréal, Quebec, Canada $(44,80)$. PMNs from healthy human donors $\left(1 \times 10^{6} \mathrm{cells} / \mathrm{ml}\right)$ were treated with PMA $(20 \mathrm{nM})$, glucose oxidase $(2 \mathrm{U} / \mathrm{ml})$, or P. aeruginosa PA14 (10 MOI) for 1 hour at $37^{\circ} \mathrm{C}$. Soluble and insoluble protein extracts were prepared as previously described (81), protein concentration was measured by the Pirce BCA Protein Assay Kit (ThermoFisher Scientific), and BPI was detected by immunoblot in $20 \mu \mathrm{g}$ of protein extract, using a mouse monoclonal anti-human BPI antibody specific for the epitope mapping between aa 227 and aa 254, i.e., the elastase cleavage site of BPI C-terminus $(1: 1,000)$ and goat anti-mouse peroxidase-labeled secondary antibody $(1: 100,000)$.

Statistics. Data were analyzed using GraphPad Prism 6 software (GraphPad Software Inc.). Two-tailed Student's $t$ test, with Welch's correction, was applied to determine the significance in the difference between two data sets. One-way ANOVA, followed by Bonferroni post-hoc, was used to compare means between multiple data sets. Pearson coefficient correlation analysis was used to determine the strength of the relationship between two parameters. $P$ values of less than 0.05 were considered significant.

Study approval. The Dartmouth College Committee for the Protection of Human Subjects (CPHS) approved the use of patient material for this study. Written informed consent was obtained from patients, and patient samples were deidentified.

\section{Author contributions}

SS and WFCR designed the research studies. AA and AHG provided the patient samples and critically reviewed the manuscript. SS and BJH conducted the experiments. MLY and MM performed proteomic analyses. SS and BJH acquired and analyzed the data. JDJ assisted with data analysis and critically reviewed the manuscript. SS and WFCR wrote the manuscript.

\section{Acknowledgments}

Research reported in this publication was supported by the National Institute of General Medical Sciences of the National Institutes of Health under award P30GM106394. We thank Brent Berwin, Iviana Torres, Sally Demirdjian, Deborah Hogan, John Hammond, Dao Nguyen, and Geoffrey McKay for providing bacterial strains and for their microbiology expertise. We thank the Translational Research Core at the Dartmouth Geisel School of Medicine. and Amanda Nymon for procuring and handling the CF samples. Microscopy was carried out at the Norris Cotton Cancer Center Microscopy Shared Resources facility with the help of Radu Stan and Kenneth Orndorff. We also thank Yina Huang for her invaluable contributions to the preparation of this manuscript.

Address correspondence to: William F.C. Rigby, One Medical Center Drive, Lebanon, New Hampshire 03756, USA. Phone: 603.650.7912; E-mail: william.f.c.rigby@dartmouth.edu.

1. O'Sullivan BP, Freedman SD. Cystic fibrosis. Lancet. 2009;373(9678):1891-1904

2. Folkesson A, et al. Adaptation of Pseudomonas aeruginosa to the cystic fibrosis airway: an evolutionary perspective. Nat Rev Microbiol. 2012;10(12):841-851.

3. Vankeerberghen A, Cuppens H, Cassiman JJ. The cystic fibrosis transmembrane conductance regulator: an intriguing protein with pleiotropic functions. J Cyst Fibros. 2002;1(1):13-29.

4. Henke MO, Ratjen F. Mucolytics in cystic fibrosis. Paediatr Respir Rev. 2007;8(1):24-29.

5. Chmiel JF, Davis PB. State of the art: why do the lungs of patients with cystic fibrosis become infected and why can't they clear the infection? Respir Res. 2003;4:8.

6. Hart CA, Winstanley C. Persistent and aggressive bacteria in the lungs of cystic fibrosis children. Br Med Bull. 2002;61:81-96.

7. Eber E, Zach MS. Pseudomonas aeruginosa infection in cystic fibrosis: prevent, eradicate or both? Thorax. 2010;65(10):849-851.

8. Lyczak JB, Cannon CL, Pier GB. Lung infections associated with cystic fibrosis. Clin Microbiol Rev. 2002;15(2):194-222.

9. Kelly E, Greene CM, McElvaney NG. Targeting neutrophil elastase in cystic fibrosis. Expert Opin Ther Targets. 2008;12(2):145-157.

10. Cheng OZ, Palaniyar N. NET balancing: a problem in inflammatory lung diseases. Front Immunol. 2013;4:1.

11. Pier GB. CFTR mutations and host susceptibility to Pseudomonas aeruginosa lung infection. Curr Opin Microbiol. 2002;5(1):81-86.

12. Gibson RL, Burns JL, Ramsey BW. Pathophysiology and management of pulmonary infections in cystic fibrosis. Am J Respir Crit Care Med. 2003;168(8):918-951.

13. Schaedel C, et al. Predictors of deterioration of lung function in cystic fibrosis. Pediatr Pulmonol. 2002;33(6):483-491.

14. Schultz H. From infection to autoimmunity: a new model for induction of ANCA against the bactericidal/permeability increasing protein (BPI). Autoimmun Rev. 2007;6(4):223-227.

15. Schultz H, Weiss J, Carroll SF, Gross WL. The endotoxin-binding bactericidal/permeability-increasing protein (BPI): a target antigen of autoantibodies. J Leukoc Biol. 2001;69(4):505-512. 
16. Aichele D, Schnare M, Saake M, Röllinghoff M, Gessner A. Expression and antimicrobial function of bactericidal permeability-increasing protein in cystic fibrosis patients. Infect Immun. 2006;74(8):4708-4714.

17. Elsbach P. The bactericidal/permeability-increasing protein (BPI) in antibacterial host defense. J Leukoc Biol. 1998;64(1):14-18

18. Kaplan MJ. Role of neutrophils in systemic autoimmune diseases. Arthritis Res Ther. 2013;15(5):219.

19. Brinkmann V, Zychlinsky A. Neutrophil extracellular traps: is immunity the second function of chromatin? J Cell Biol. 2012;198(5):773-783.

20. Jennette JC, Falk RJ. Pathogenesis of antineutrophil cytoplasmic autoantibody-mediated disease. Nat Rev Rheumatol. 2014;10(8):463-473.

21. Demoruelle MK, Solomon JJ, Fischer A, Deane KD. The lung may play a role in the pathogenesis of rheumatoid arthritis. Int $J$ Clin Rheumtol. 2014;9(3):295-309.

22. Pruijn GJ. Citrullination and carbamylation in the pathophysiology of rheumatoid arthritis. Front Immunol. $2015 ; 6: 192$.

23. Catrina AI, Ytterberg AJ, Reynisdottir G, Malmström V, Klareskog L. Lungs, joints and immunity against citrullinated proteins in rheumatoid arthritis. Nat Rev Rheumatol. 2014;10(11):645-653.

24. Challener GJ, et al. Anti-carbamylated protein antibody levels correlate with anti-Sa (citrullinated vimentin) antibody levels in rheumatoid arthritis. J Rheumatol. 2016;43(2):273-281.

25. Humphreys $\mathrm{JH}$, et al. Anticarbamylated protein antibodies are associated with long-term disability and increased disease activity in patients with early inflammatory arthritis: results from the Norfolk Arthritis Register. Ann Rheum Dis. 2016;75(6):1139-1144.

26. Shi J, et al. The specificity of anti-carbamylated protein antibodies for rheumatoid arthritis in a setting of early arthritis. Arthritis Res Ther. 2015;17:339

27. Green NM, Marshak-Rothstein A. Toll-like receptor driven B cell activation in the induction of systemic autoimmunity. Semin Immunol. 2011;23(2):106-112.

28. Gray RD, McCullagh BN, McCray PB. NETs and CF Lung Disease: Current Status and Future Prospects. Antibiotics (Basel). 2015;4(1):62-75.

29. Marcos V, et al. Free DNA in cystic fibrosis airway fluids correlates with airflow obstruction. Mediators Inflamm. 2015;2015:408935.

30. Dwyer M, et al. Cystic fibrosis sputum DNA has NETosis characteristics and neutrophil extracellular trap release is regulated by macrophage migration-inhibitory factor. J Innate Immun. 2014;6(6):765-779.

31. Milla CE, Warwick WJ. Risk of death in cystic fibrosis patients with severely compromised lung function. Chest. 1998;113(5):1230-1234.

32. Kerem E, et al. Factors associated with FEV1 decline in cystic fibrosis: analysis of the ECFS patient registry. Eur Respir J. 2014;43(1):125-133.

33. Elizur A, Cannon CL, Ferkol TW. Airway inflammation in cystic fibrosis. Chest. 2008;133(2):489-495.

34. Rao S, Grigg J. New insights into pulmonary inflammation in cystic fibrosis. Arch Dis Child. 2006;91(9):786-788.

35. Chen DL, Ferkol TW, Mintun MA, Pittman JE, Rosenbluth DB, Schuster DP. Quantifying pulmonary inflammation in cystic fibrosis with positron emission tomography. Am J Respir Crit Care Med. 2006;173(12):1363-1369.

36. Cutting GR. Cystic fibrosis genetics: from molecular understanding to clinical application. Nat Rev Genet. 2015;16(1):45-56.

37. Schechter MS. Nongenetic influences on cystic fibrosis outcomes. Curr Opin Pulm Med. 2011;17(6):448-454.

38. Bruscia EM, Bonfield TL. Innate and adaptive immunity in cystic fibrosis. Clin Chest Med. 2016;37(1):17-29.

39. Xu Y, Worgall S. Immune Dysfunction in Cystic Fibrosis. In: Sriramulu D, ed. Cystic Fibrosis - Renewed Hopes Through Research. InTech; 2012: 273-288.

40. Chmiel JF, Davis PB. State of the art: why do the lungs of patients with cystic fibrosis become infected and why can't they clear the infection? Respir Res. 2003;4:8.

41. Shi J, et al. Anti-carbamylated protein (anti-CarP) antibodies precede the onset of rheumatoid arthritis. Ann Rheum Dis. 2014;73(4):780-783.

42. Schultz H, Hume J, Zhang DS, Gioannini TL, Weiss JP. A novel role for the bactericidal/permeability increasing protein in interactions of gram-negative bacterial outer membrane blebs with dendritic cells. J Immunol. 2007;179(4):2477-2484

43. Gray PW, et al. Cloning of the cDNA of a human neutrophil bactericidal protein. Structural and functional correlations. $J$ Biol Chem. 1989;264(16):9505-9509.

44. LaFayette SL, et al. Cystic fibrosis-adapted Pseudomonas aeruginosa quorum sensing lasR mutants cause hyperinflammatory responses. Sci Adv. 2015;1(6):e1500199.

45. Pritt B, O'Brien L, Winn W. Mucoid Pseudomonas in cystic fibrosis. Am J Clin Pathol. 2007;128(1):32-34.

46. Moss RB. Hypergammaglobulinemia in cystic fibrosis. Role of Pseudomonas endobronchial infection. Chest. 1987;91(4):522-526.

47. Khandpur R, et al. NETs are a source of citrullinated autoantigens and stimulate inflammatory responses in rheumatoid arthritis. Sci Transl Med. 2013;5(178):178ra40.

48. Rahman S, Gadjeva M. Does NETosis contribute to the bacterial pathoadaptation in cystic fibrosis? Front Immunol. $2014 ; 5: 378$.

49. Urban CF, Reichard U, Brinkmann V, Zychlinsky A. Neutrophil extracellular traps capture and kill Candida albicans yeast and hyphal forms. Cell Microbiol. 2006;8(4):668-676.

50. Young RL, et al. Neutrophil extracellular trap (NET)-mediated killing of Pseudomonas aeruginosa: evidence of acquired resistance within the CF airway, independent of CFTR. PLoS ONE. 2011;6(9):e23637.

51. Lundström E, Källberg H, Alfredsson L, Klareskog L, Padyukov L. Gene-environment interaction between the DRB1 shared epitope and smoking in the risk of anti-citrullinated protein antibody-positive rheumatoid arthritis: all alleles are important. Arthritis Rheum. 2009;60(6):1597-1603.

52. Demoruelle MK, et al. Brief report: airways abnormalities and rheumatoid arthritis-related autoantibodies in subjects without arthritis: early injury or initiating site of autoimmunity? Arthritis Rheum. 2012;64(6):1756-1761.

53. Cooper T, et al. Clinical associations and characterisation of antineutrophil cytoplasmic antibodies directed against bactericidal/permeability-increasing protein and azurocidin. Rheumatol Int. 2000;19(4):129-136.

54. MacEachran DP, et al. The Pseudomonas aeruginosa secreted protein PA2934 decreases apical membrane expression of the cystic fibrosis transmembrane conductance regulator. Infect Immun. 2007;75(8):3902-3912. 
55. Swiatecka-Urban A, et al. Pseudomonas aeruginosa inhibits endocytic recycling of CFTR in polarized human airway epithelial cells. Am J Physiol, Cell Physiol. 2006;290(3):C862-C872.

56. Bomberger JM, et al. Pseudomonas aeruginosa Cif protein enhances the ubiquitination and proteasomal degradation of the transporter associated with antigen processing (TAP) and reduces major histocompatibility complex (MHC) class I antigen presentation. J Biol Chem. 2014;289(1):152-162.

57. Castellani C, et al. Consensus on the use and interpretation of cystic fibrosis mutation analysis in clinical practice. J Cyst Fibros 2008;7(3):179-196.

58. Ratner D, Mueller C. Immune responses in cystic fibrosis: are they intrinsically defective? Am J Respir Cell Mol Biol. 2012;46(6):715-722.

59. Holoshitz J. The rheumatoid arthritis HLA-DRB1 shared epitope. Curr Opin Rheumatol. 2010;22(3):293-298.

60. Jiang X, et al. Anti-CarP antibodies in two large cohorts of patients with rheumatoid arthritis and their relationship to genetic risk factors, cigarette smoking and other autoantibodies. Ann Rheum Dis. 2014;73(10):1761-1768.

61. Herrod HG, Amos DB, Spock A. HLA determinants in families with more than one member with cystic fibrosis. $J$ Pediatr. 1979;94(4):676.

62. Slieker MG, Sanders EA, Rijkers GT, Ruven HJ, van der Ent CK. Disease modifying genes in cystic fibrosis. J Cyst Fibros. 2005;4 Suppl 2:7-13.

63. Mahadeva R, et al. Anti-neutrophil cytoplasmic antibodies (ANCA) against bactericidal/permeability-increasing protein (BPI) and cystic fibrosis lung disease. Clin Exp Immunol. 1999;117(3):561-567.

64. Schultz H, Heintz H, van Zandbergen G, Ullrich S, Reinhold-Keller E, Gross WL. ANCA against the bactericidal/permeability increasing protein (BPI-ANCA) can compromise the antibiotic function of BPI in a Wegener's granulomatosis patient. Clin Exp Rheumatol. 2003;21(6):763-766.

65. Britigan BE, Hayek MB, Doebbeling BN, Fick RB. Transferrin and lactoferrin undergo proteolytic cleavage in the Pseudomonas aeruginosa-infected lungs of patients with cystic fibrosis. Infect Immun. 1993;61(12):5049-5055.

66. Schmidtchen A, Frick IM, Andersson E, Tapper H, Björck L. Proteinases of common pathogenic bacteria degrade and inactivate the antibacterial peptide LL-37. Mol Microbiol. 2002;46(1):157-168.

67. Downey DG, Bell SC, Elborn JS. Neutrophils in cystic fibrosis. Thorax. 2009;64(1):81-88.

68. Brink M, et al. Anti-carbamylated protein antibodies in the pre-symptomatic phase of rheumatoid arthritis, their relationship with multiple anti-citrulline peptide antibodies and association with radiological damage. Arthritis Res Ther. 2015;17:25.

69. Bergum B, et al. Antibodies against carbamylated proteins are present in primary Sjögren's syndrome and are associated with disease severity. Ann Rheum Dis. 2016;75(8):1494-1500

70. Sedivá A, et al. Antineutrophil cytoplasmic autoantibodies (ANCA) in children with cystic fibrosis. J Autoimmun. 1998;11(2):185-190.

71. Whiteson $\mathrm{KL}$, et al. Breath gas metabolites and bacterial metagenomes from cystic fibrosis airways indicate active $\mathrm{pH}$ neutral 2,3-butanedione fermentation. ISME J. 2014;8(6):1247-1258.

72. Ercolini AM, Miller SD. The role of infections in autoimmune disease. Clin Exp Immunol. 2009;155(1):1-15.

73. Sfriso P, et al. Infections and autoimmunity: the multifaceted relationship. J Leukoc Biol. 2010;87(3):385-395.

74. Wada Y, Kuroda T, Murasawa A, Nakano M, Narita I. Anti-neutrophil cytoplasmic autoantibodies against bactericidal/permeability-increasing protein in patients with rheumatoid arthritis and their correlation with bronchial involvement. Mod Rheumatol. 2010;20(3):252-256

75. Dhasmana DJ, Wilson R. Bronchiectasis and autoimmune disease. In: Floto RA, Haworth CS, eds. Bronchiectasis. European Respiratory Society; 2011:192-210.

76. Brezski RJ, Jordan RE. Cleavage of IgGs by proteases associated with invasive diseases: an evasion tactic against host immunity? MAbs. 2010;2(3):212-220.

77. Folds JD, Prince H, Spitznagel JK. Limited cleavage of human immunoglobulins by elastase of human neutrophil polymorphonuclear granulocytes. Possible modulator of immune complex disease. Lab Invest. 1978;39(4):313-321.

78. Brinkmann V, Laube B, Abu Abed U, Goosmann C, Zychlinsky A. Neutrophil extracellular traps: how to generate and visualize them. $J$ Vis Exp. 2010;(36):1724.

79. Yang H, Zubarev RA. Mass spectrometric analysis of asparagine deamidation and aspartate isomerization in polypeptides. Electrophoresis. 2010;31(11):1764-1772.

80. Hammond JH, Dolben EF, Smith TJ, Bhuju S, Hogan DA. Links between Anr and Quorum Sensing in Pseudomonas aeruginosa Biofilms. J Bacteriol. 2015;197(17):2810-2820.

81. Hamilton BJ, Nagy E, Malter JS, Arrick BA, Rigby WF. Association of heterogeneous nuclear ribonucleoprotein A1 and C proteins with reiterated AUUUA sequences. J Biol Chem. 1993;268(12):8881-8887. 